\title{
Explicit Calculation of Some Polynomials Introduced by W. Gautschi
}

\author{
H.-J. Fischer
}

\begin{abstract}
In his classical by now investigation of the numerical condition of methods for computing orthogonal polynomials from modified moments, Gautschi introduced some polynomials defined by the nodes and weights of a Gaussian quadrature formula for the measure of orthogonality. We show that these polynomials can be calculated explicitly for Chebyshev weights of first, second and third kinds.
\end{abstract}

Keywords: Numerical condition, orthogonal polynomials, modified moments, Chebyshev and Jacobi weights

AMS subject classification: $42 \mathrm{C} 05$

\section{Introduction}

The polynomials in question were introduced by Gautschi when he analyzed the numerical condition of the method of modified moments: Let $\sigma$ be a given positive measure with infinite support $S(\sigma)$. Then there uniquely exists a family of monic polynomials $\pi_{j}$ with

$$
\int \pi_{l}(x) \pi_{j}(x) d \sigma(x)=0 \quad(l<j) \quad \text { and } \quad \int \pi_{j}^{2}(x) d \sigma(x)>0 \quad(j \geq 0)
$$

They satisfy a three-term recurrence relation

$$
\pi_{j+1}(x)=\left(x-\alpha_{j}\right) \pi_{j}(x)-\beta_{j} \pi_{j-1}(x) \quad(j \geq 0)
$$

if we set $\pi_{-1}(x) \equiv 0$. It is well known that the generation of the orthogonal polynomials (or, equivalently, of the coefficients $\alpha_{j}$ and $\beta_{j}$ ) from ordinary moments

$$
\mu_{k}=\int x^{k} d \sigma(x)
$$

is severely ill-conditioned (see the classical paper of Gautschi [4]). More promising are modified moments

$$
m_{k}=\int q_{k}(x) d \sigma(x)
$$

H.-J. Fischer: Techn. Univ. Chemnitz, Fak. Math., D-09107 Chemnitz

ISSN 0232-2064 / \$2.50 (C) Heldermann Verlag Berlin 
with some properly chosen set of polynomials $q_{k}$ (e.g., Chebyshev polynomials or other polynomials orthogonal with respect to some measure $s$ ). It would be desirable to calculate or estimate the condition of the map $K_{n}$ from $m=\left[m_{0}, \ldots, m_{2 n-1}\right]^{T}$ to $\rho=\left[\alpha_{0}, \ldots, \alpha_{n-1}, \beta_{0}, \ldots, \beta_{n-1}\right]^{T}$. In the papers $[5,6]$ Gautschi introduced the vector

$$
\gamma=\left[\sigma_{1}, \ldots, \sigma_{n}, \tau_{1}, \ldots, \tau_{n}\right]^{T}
$$

of weights and nodes of the Gauss-Christoffel quadrature rule

$$
\int q(x) d \sigma(x)=\sum_{k=1}^{n} \sigma_{k} q\left(\tau_{k}\right)
$$

for any polynomial $q$ of degree less or equal $2 n-1$. He denoted by $G_{n}$ the map

$$
G_{n}: \mathbb{R}^{2 n} \rightarrow \mathbb{R}^{2 n}, \quad m \rightarrow \gamma
$$

and by $H_{n}$ the (well-conditioned) map

$$
H_{n}: \mathbb{R}^{2 n} \rightarrow \mathbb{R}^{2 n}, \quad \gamma \rightarrow \rho .
$$

Then one has the decomposition

$$
K_{n}=H_{n} \circ G_{n},
$$

and this equation reduces our task to the investigation of the map $G_{n}$. More precisely, Gautschi considered the map defined by

$$
\widetilde{G}_{n}: \mathbb{R}^{2 n} \rightarrow \mathbb{R}^{2 n}, \quad \tilde{m} \rightarrow \gamma
$$

where

$$
\tilde{m}_{k}=d_{k}^{-\frac{1}{2}} m_{k} \quad \text { with } \quad d_{k}=\int q_{k}^{2}(x) d s(x) .
$$

The map $\widetilde{G}_{n}$ differs from $G_{n}$ only by a trivial diagonal map, and the sensitivity of the nonlinear map $\widetilde{G}_{n}$ can be measured by the norm of the Fréchet derivative $\widetilde{G}_{n}^{\prime}$, which is a linear map (the Jacobian of $\widetilde{G}_{n}$ ) from $\mathbb{R}^{2 n}$ into $\mathbb{R}^{2 n}$. We consider here only the Frobenius norm

$$
\|A\|_{F}=\left(\operatorname{Tr} A^{T} A\right)^{\frac{1}{2}}=\left(\sum_{j, k=0}^{2 n-1} a_{j k}^{2}\right)^{\frac{1}{2}} .
$$

In the paper [2], Gautschi proved the equation

$$
\left\|\tilde{G}_{n}^{\prime}\right\|_{F}=\left\{\int g_{n}(x) d s(x)\right\}^{\frac{1}{2}}
$$

with some polynomial $g_{n}$ of degree $4 n-2$ determined by $\gamma$, which will be defined in the following section (this is essentially equation (3.2) of [6], though the result is implicitly contained already in the proof of Theorem 3.1 of the article [5]).

The present paper is devoted to the explicit calculation of the polynomials $g_{n}$ in some special cases. For a direct approach to the investigation of the map $K_{n}$, see the article [2] of the author. The map $H_{n}$ is investigated in the subsequent papers of Gautschi $[5-8]$ and in the article [3] of the author. 


\section{Polynomial interpolation}

The polynomials $g_{n}$ are defined as follows:

$$
g_{n}(x)=\sum_{j=1}^{n} h_{j}^{2}(x)+\sum_{j=1}^{n} \sigma_{j}^{-2} k_{j}^{2}(x)
$$

where $\sigma_{j}$ are the weights of the Gauss-Christoffel quadrature rule, and $h_{j}$ and $k_{j}$ are the fundamental polynomials of Hermite interpolation in the nodes of the quadrature rule. We define them after recalling the Lagrange interpolation. Let $\left\{\tau_{i}\right\}_{i=1, \ldots, n}$ be a set of distinct numbers, and define

$$
l_{i}(x)=\frac{\omega_{n}(x)}{\omega_{n}^{\prime}\left(\tau_{i}\right)\left(x-\tau_{i}\right)} \quad(i=1, \ldots, n),
$$

where $\omega_{n}(x)=\left(x-\tau_{1}\right) \cdots\left(x-\tau_{n}\right)$. The Lagrange interpolation formula

$$
q(x)=\sum_{i=1}^{n} q\left(\tau_{i}\right) l_{i}(x) \quad\left(q \in \mathcal{P}_{n-1}\right)
$$

is well known. The following result is well known, too.

Lemma 1. Let

$$
h_{i}(x)=\left[1-\frac{\omega_{n}^{\prime \prime}\left(\tau_{i}\right)}{\omega_{n}^{\prime}\left(\tau_{i}\right)}\left(x-\tau_{i}\right)\right] l_{i}^{2}(x) \quad \text { and } \quad k_{i}(x)=\left(x-\tau_{i}\right) l_{i}^{2}(x) .
$$

Then we have the equation

$$
q(x)=\sum_{i=1}^{n} q\left(\tau_{i}\right) h_{i}(x)+\sum_{i=1}^{n} q^{\prime}\left(\tau_{i}\right) k_{i}(x) \quad\left(q \in \mathcal{P}_{2 n-1}\right)
$$

In our case, the nodes $\tau_{i}$ are the zeros of some orthogonal polynomial $p_{n}$. We remark here that in equations (3) and (5) we can use the (generally not monic) polynomial $p_{n}$ instead of $\omega_{n}$, since the leading coefficient will simply cancel out.

In the following, we will need an elementary interpolation identity of higher order.

Lemma 2. Let $\left\{\tau_{i}\right\}_{i=1, \ldots, n}$ be any set of distinct nodes, and let $b_{i} \in \mathcal{P}_{4 n-1}$ be polynomials with the property

$$
b_{i}\left(\tau_{j}\right)=\delta_{i j} \quad \text { and } \quad b_{i}^{\prime}\left(\tau_{j}\right)=b_{i}^{\prime \prime}\left(\tau_{j}\right)=b_{i}^{\prime \prime \prime}\left(\tau_{j}\right)=0 \quad(i, j=1, \ldots, n) .
$$

Then the identity $\sum_{i=1}^{n} b_{i}(x) \equiv 1$ is satisfied.

Proof. Under our assumptions the polynomial $r \in \mathcal{P}_{4 n-1}$,

$$
r(x)=\sum_{i=1}^{n} b_{i}(x)-1
$$

vanishes at all nodes $\tau_{i}$ together with all derivatives up to the third, and thus it is divisible by the polynomial $\left(x-\tau_{1}\right)^{4} \cdots\left(x-\tau_{n}\right)^{4}$ having degree $4 n$. Consequently, the polynomial $r$ must be identically zero 
In fact, the polynomials $b_{i}$ are well defined by the above properties and can be written down explicitly in terms of the polynomials $l_{i}$.

Lemma 3. Let $l_{i}$ be the fundamental polynomials of Lagrange interpolation for the nodes $\left\{\tau_{i}\right\}_{i=1, \ldots, n}$ and let the first few coefficients of the Taylor expansion of $l_{i}$ around $\tau_{i}$ be

$$
l_{i}(x)=1+c_{1}\left(\tau_{i}\right)\left(x-\tau_{i}\right)+c_{2}\left(\tau_{i}\right)\left(x-\tau_{i}\right)^{2}+c_{3}\left(\tau_{i}\right)\left(x-\tau_{i}\right)^{3}+\ldots
$$

Then we have

$$
b_{i}(x)=\left[1+d_{1}\left(\tau_{i}\right)\left(x-\tau_{i}\right)+d_{2}\left(\tau_{i}\right)\left(x-\tau_{i}\right)^{2}+d_{3}\left(\tau_{i}\right)\left(x-\tau_{i}\right)^{3}\right] l_{i}^{4}(x),
$$

where the coefficients $d_{1}, d_{2}, d_{3}$ can be obtained from

$$
\begin{aligned}
& d_{1}=-4 c_{1} \\
& d_{2}=10 c_{1}^{2}-4 c_{2} \\
& d_{3}=-20 c_{1}^{3}+20 c_{1} c_{2}-4 c_{3} .
\end{aligned}
$$

Proof. If

$$
1+d_{1}\left(\tau_{i}\right)\left(x-\tau_{i}\right)+d_{2}\left(\tau_{i}\right)\left(x-\tau_{i}\right)^{2}+d_{3}\left(\tau_{i}\right)\left(x-\tau_{i}\right)^{3}
$$

coincides with the first few terms of the Taylor series of $l_{i}(x)^{-4}$, then $b_{i}$ as defined in Lemma 3 satisfies $b_{i}(x)=1+O\left(\left(x-\tau_{i}\right)^{4}\right)$. Since $b_{i}$ clearly has degree less or equal $4 n-1$ and vanishes at each $\tau_{j} \neq \tau_{i}$, it follows that it is identical with $b_{i}$ defined in Lemma 2. The Taylor series can be obtained easily: From

$$
\sum_{j=0}^{\infty} d_{j}\left(x-\tau_{i}\right)^{j}=\left(\sum_{j=0}^{\infty} c_{j}\left(x-\tau_{i}\right)^{j}\right)^{-4}
$$

by differentiating and multiplying by $\sum_{j=0}^{\infty} c_{j}\left(x-\tau_{i}\right)^{j}$ we have the equation

$$
\sum_{j=1}^{\infty} j d_{j}\left(x-\tau_{i}\right)^{j-1} \sum_{j=0}^{\infty} c_{j}\left(x-\tau_{i}\right)^{j}=-4 \sum_{j=1}^{\infty} j c_{j}\left(x-\tau_{i}\right)^{j-1} \sum_{j=0}^{\infty} d_{j}\left(x-\dot{\tau}_{i}\right)^{j}
$$

A comparison of the coefficients gives the linear system

$$
\begin{aligned}
d_{1} & =-4 c_{1} \\
c_{1} d_{1}+2 d_{2} & =-4\left(c_{1} d_{1}+2 c_{2}\right) \\
c_{2} d_{1}+2 c_{1} d_{2}+3 d_{3} & =-4\left(c_{1} d_{2}+2 c_{2} d_{1}+3 c_{3}\right)
\end{aligned}
$$

and solving for $d_{j}$ we arrive at our proposition 


\section{Explicit calculation of $\sum_{i=1}^{n} \sigma_{i}^{-2} k_{i}^{2}(x)$ for Jacobi polynomials}

Of course, we will be able to compute explicit formulas only in some special cases. The Jacobi polynomials $P_{n}^{(\alpha, \beta)}$ are orthogonal with respect to the measure defined by

$$
d \sigma(x)=(1-x)^{\alpha}(1+x)^{\beta} d x
$$

their leading coefficient (in their traditional standardization as in [1: Formula 22.2.1] is

$$
K_{n}=\frac{1}{2^{n}}\left(\begin{array}{c}
2 n+\alpha+\beta \\
n
\end{array}\right)
$$

and the constant in their orthogonality relation is

$$
H_{n}=\frac{2^{\alpha+\beta+1}}{2 n+\alpha+\beta+1} \frac{\Gamma(n+\alpha+1) \Gamma(n+\beta+1)}{n ! \Gamma(n+\alpha+\beta+1)} .
$$

We will write simply $p_{n}$ instead of $P_{n}^{(\alpha, \beta)}$. The three-term recurrence for $p_{n}$ has the explicit form

$$
\begin{aligned}
2 n(n+ & \alpha+\beta)(2 n+\alpha+\beta-2) p_{n}(x) \\
= & {\left[(2 n+\alpha+\beta-1)\left(\alpha^{2}-\beta^{2}\right)\right.} \\
& +(2 n+\alpha+\beta-2)(2 n+\alpha+\beta-1)(2 n+\alpha+\beta) x] p_{n-1}(x) \\
& -2(n+\alpha-1)(n+\beta-1)(2 n+\alpha+\beta) p_{n-2}(x) .
\end{aligned}
$$

For these special polynomials we have some more useful relations. Especially, they satisfy the differential equation

$$
\left(1-x^{2}\right) p_{n}^{\prime \prime}(x)+[\beta-\alpha-(\alpha+\beta+2) x] p_{n}^{\prime}(x)+n(n+\alpha+\beta+1) p_{n}(x)=0,
$$

and their derivatives can be expressed as

$$
\begin{aligned}
& (2 n+\alpha+\beta)\left(1-x^{2}\right) p_{n}^{\prime}(x) \\
& \quad=n[\alpha-\beta-(2 n+\alpha+\beta) x] p_{n}(x)+2(n+\alpha)(n+\beta) p_{n-1}(x)
\end{aligned}
$$

Now we can prove the following

Theorem 1. Let $\tau_{i}$ be the zeros of the Jacobi polynomial $p_{n}$. Then the polynomials $k_{i}$ defined in equations (5) satisfy the identity

$$
\sum_{i=1}^{n} \sigma_{i}^{-2} k_{i}^{2}(x)=C_{n}^{2} p_{n}^{2}(x)\left[p_{n-1}^{2}(x)-B_{n} p_{n-2}(x) p_{n}(x)\right]
$$


with the constants

$$
C_{n}=\frac{K_{n-1}}{K_{n} H_{n-1}} \quad \text { and } \quad B_{n}=\frac{(2 n+\alpha+\beta)(n+\alpha-1)(n+\beta-1)}{(2 n+\alpha+\beta-1)(n+\alpha)(n+\beta)} .
$$

Proof. For any set of orthogonal polynomials the weights of Gauss-Christoffel quadrature formulas can be written as

$$
\sigma_{i}^{-1}=C_{n} p_{n}^{\prime}\left(\tau_{i}\right) p_{n-1}\left(\tau_{i}\right) \text {. }
$$

From the definition (5) of the polynomials $k_{i}$ and $l_{i}(x)=\frac{p_{n}(x)}{\left(x-\tau_{i}\right) p_{n}^{\prime}\left(r_{i}\right)}$ we obtain easily

$$
\sum_{i=1}^{n} \sigma_{i}^{-2} k_{i}^{2}(x)=C_{n}^{2} p_{n}^{2}(x) \sum_{i=1}^{n} p_{n-1}^{2}\left(\tau_{i}\right) l_{i}^{2}(x)
$$

The sum on the right-hand side can be evaluated from the interpolation identity (6). First we observe that from the differential equation the explicit formula

$$
h_{i}(x)=\left[1-\frac{(\alpha+\beta+2) \tau_{i}+\alpha-\beta}{1-\tau_{i}^{2}}\left(x-\tau_{i}\right)\right] l_{i}^{2}(x)
$$

can be derived. Thus equation (6) can be written as

$$
\sum_{i=1}^{n} q\left(\tau_{i}\right) l_{i}^{2}(x)=q(x)-\sum_{i=1}^{n}\left[q^{\prime}\left(\tau_{i}\right)-\frac{(\alpha+\beta+2) \tau_{i}+\alpha-\beta}{1-\tau_{i}^{2}} q\left(\tau_{i}\right)\right]\left(x-\tau_{i}\right) l_{i}^{2}(x)
$$

for any polynomial $q \in \mathcal{P}_{2 n-1}$. Now we set $q=p_{n-1}^{2}$. Since we have

$$
\left(x-\tau_{i}\right) l_{i}(x)=\frac{1}{p_{n}^{\prime}\left(\tau_{i}\right)} p_{n}(x)
$$

and from equation (6)

$$
p_{n}^{\prime}\left(\tau_{i}\right)=\frac{2(n+\alpha)(n+\beta)}{(2 n+\alpha+\beta)\left(1-\tau_{i}^{2}\right)} p_{n-1}\left(\tau_{i}\right)
$$

we obtain

$$
\begin{aligned}
& {\left[q^{\prime}\left(\tau_{i}\right)-\frac{(\alpha+\beta+2) \tau_{i}+\alpha-\beta}{1-\tau_{i}^{2}} q\left(\tau_{i}\right)\right] \frac{1}{p_{n}^{\prime}\left(\tau_{i}\right)}} \\
& \quad=\frac{2 n+\alpha+\beta}{(n+\alpha)(n+\beta)}\left\{\left(1-\tau_{i}^{2}\right) p_{n-1}^{\prime}\left(\tau_{i}\right)-\frac{1}{2}\left[(\alpha+\beta+2) \tau_{i}+\alpha-\beta\right] p_{n-1}\left(\tau_{i}\right)\right\} .
\end{aligned}
$$

If we express $\left(1-\tau_{i}^{2}\right) p_{n-1}^{\prime}\left(\tau_{i}\right)$ using $(9)$ and eliminate the terms $\tau_{i} p_{n-1}\left(\tau_{i}\right)$ using (7), after an easy (but tedious) calculation we arrive at

$$
\left(1-\tau_{i}^{2}\right) p_{n-1}^{\prime}\left(\tau_{i}\right)-\frac{1}{2}\left[(\alpha+\beta+2) \tau_{i}+\alpha-\beta\right] p_{n-1}\left(\tau_{i}\right)=\frac{(n+\alpha-1)(n+\beta-1)}{2 n+\alpha+\beta-1} p_{n-2}\left(\tau_{i}\right) .
$$

This implies

$$
\sum_{i=1}^{n}\left[q^{\prime}\left(\tau_{i}\right)-\frac{(\alpha+\beta+2) \tau_{i}+\alpha-\beta}{1-\tau_{i}^{2}} q\left(\tau_{i}\right)\right]\left(x-\tau_{i}\right) l_{i}^{2}(x)=B_{n} p_{n}(x) \sum_{i=1}^{n} p_{n-2}\left(\tau_{i}\right) l_{i}(x),
$$

and the sum on the right clearly is equal to $p_{n-2}(x)$ due to the Lagrange interpolation formula (4) 
Since we are able to obtain further results in the special case of Chebyshev polynomials, where $\alpha, \beta= \pm \frac{1}{2}$, and the traditional standardization of Chebyshev polynomials is different from that of Jacobi polynomials, we reformulate our result in this case. While Chebyshev polynomials of first and second kinds are well known, the nomenclature for the mixed cases $(\alpha=-\beta)$ was introduced by Gautschi (e.g., in [9]) and adopted by other authors (in the papers $[10,11]$ of Mason and Elliott we can find some properties of Chebyshev polynomials of third and fourth kinds).

First we recall the connection between these special Jacobi polynomials and Chebyshev polynomials (see equations $22.5 .30-22.5 .32$ in [1]):

$$
\begin{aligned}
T_{n}(x) & =\frac{n ! \sqrt{\pi}}{\Gamma\left(n+\frac{1}{2}\right)} P_{n}^{\left(-\frac{1}{2},-\frac{1}{2}\right)}(x) \\
U_{n}(x) & =\frac{(n+1) ! \sqrt{\pi}}{2 \Gamma\left(n+\frac{3}{2}\right)} P_{n}^{\left(\frac{1}{2}, \frac{1}{2}\right)}(x) \\
U_{2 n} & =\frac{n ! \sqrt{\pi}}{\Gamma\left(n+\frac{1}{2}\right)} P_{n}^{\left(\frac{1}{2},-\frac{1}{2}\right)}\left(2 x^{2}-1\right)
\end{aligned}
$$

We will rewrite the last equation in a more convenient way. The trigonometric representation of Chebyshev polynomials implies

$$
U_{2 n}(x)=U_{n}\left(2 x^{2}-1\right)+U_{n-1}\left(2 x^{2}-1\right)
$$

and this equation gives immediately

$$
U_{n}(x)+U_{n-1}(x)=\frac{n ! \sqrt{\pi}}{\Gamma\left(n+\frac{1}{2}\right)} P_{n}^{\left(\frac{1}{2},-\frac{1}{2}\right)}(x) .
$$

Since we have

$$
P_{n}^{\left(-\frac{1}{2}, \frac{1}{2}\right)}(x)=(-1)^{n} P_{n}^{\left(\frac{1}{2},-\frac{1}{2}\right)}(-x)
$$

we obtain easily the equation

$$
U_{n}(x)-U_{n-1}(x)=\frac{n ! \sqrt{\pi}}{\Gamma\left(n+\frac{1}{2}\right)} P_{n}^{\left(-\frac{1}{2}, \frac{1}{2}\right)}(x) .
$$

That is why we can call the polynomials $V_{n}=U_{n}-U_{n-1}$ Chebyshev polynomials of third kind and the polynomials $W_{n}=U_{n}+U_{n-1}$ Chebyshev polynomials of fourth kind. Their trigonometric representation is

$$
V_{n}(\cos \theta)=\frac{\cos \left(n+\frac{1}{2}\right) \theta}{\cos \frac{1}{2} \theta} \quad \text { and } \quad W_{n}(\cos \theta)=\frac{\sin \left(n+\frac{1}{2}\right) \theta}{\sin \frac{1}{2} \theta}
$$

(see equations (1.3) and (1.4) in the paper [10]). In view of the obvious property $W_{n}(x)=(-1)^{n} V_{n}(-x)$, there will be no need to consider the polynomials of fourth kind in the following.

Our Theorem 1 can be specialized for Chebyshev polynomials. 
Corollary 1. For Chebyshev polynomials of first, second and third kinds we have

$$
\begin{aligned}
& \sum_{i=1}^{n} \sigma_{i}^{-2} k_{i}^{2}(x)=\frac{1}{\pi^{2}}\left[1-x^{2}+\frac{3}{2 n} T_{n-2}(x) T_{n}(x)\right] T_{n}^{2}(x) \\
& \sum_{i=1}^{n} \sigma_{i}^{-2} k_{i}^{2}(x)=\frac{1}{\pi^{2}}\left[1+\frac{3}{2(n+1)} U_{n-2}(x) U_{n}(x)\right] U_{n}^{2}(x) \\
& \sum_{i=1}^{n} \sigma_{i}^{-2} k_{i}^{2}(x)=\frac{1}{4 \pi^{2}}\left[2(1-x)+\frac{3}{2 n+1} V_{n-2}(x) V_{n}(x)\right] V_{n}^{2}(x),
\end{aligned}
$$

respectively.

Proof. By simple substitution for the left-hand sum we have

$$
\begin{aligned}
& \frac{1}{\pi^{2}}\left[T_{n-1}^{2}(x)-\frac{n-\frac{3}{2}}{n} T_{n-2}(x) T_{n}(x)\right] T_{n}^{2}(x) \\
& \frac{1}{\pi^{2}}\left[U_{n-1}^{2}(x)-\frac{n-\frac{1}{2}}{n+1} U_{n-2}(x) U_{n}(x)\right] U_{n}^{2}(x) \\
& \frac{1}{4 \pi^{2}}\left[V_{n-1}^{2}(x)-\frac{n-1}{n+\frac{1}{2}} V_{n-2}(x) V_{n}(x)\right] V_{n}^{2}(x)
\end{aligned}
$$

in the first, second and third cases, respectively. Now our proposition follows at once from the elementary identities

$$
\begin{aligned}
T_{n-1}^{2}(x)-T_{n-2}(x) T_{n}(x) & =1-x^{2} \\
U_{n-1}^{2}(x)-U_{n-2}(x) U_{n}(x) & =1 \\
V_{n-1}^{2}(x)-V_{n-2}(x) V_{n}(x) & =2(1-x) .
\end{aligned}
$$

The corollary is proved

\section{An estimate of $\sum_{i=1}^{n} h_{i}^{2}(x)$ for $\alpha, \beta \in(-1,0]$}

Interestingly, the sum $\sum_{i=1}^{n} h_{i}^{2}(x)$ in this case is uniformly bounded.

Theorem 2. Let $\tau_{i}$ be the zeros of $P_{n}^{(\alpha, \beta)}$ and $\alpha, \beta \in(-1,0]$. Then the polynomials $h_{i}$ defined in (5) satisfy the inequality

$$
\sum_{i=1}^{n} h_{i}^{2}(x) \leq 1 \quad(x \in[-1,1]) .
$$

Proof. All we have to prove is the non-negativity of $h_{i}$ in $[-1,1]$ : From (6) with $q \equiv 1$ we obtain $\sum_{i=1}^{n} h_{i}(x) \equiv 1$, consequently $0 \leq h_{i}(x) \leq 1$ and

$$
\sum_{i=1}^{n} h_{i}^{2}(x) \leq \sum_{i=1}^{n} h_{i}(x) \equiv 1 .
$$


Since

$$
h_{i}(x)=\left[1-\frac{(\alpha+\beta+2) \tau_{i}+\alpha-\beta}{1-\tau_{i}^{2}}\left(x-\tau_{i}\right)\right] l_{i}^{2}(x)
$$

and all $\tau_{i} \in(-1,1)$, it is sufficient to show

$$
1-\frac{(\alpha+\beta+2) \tau+\alpha-\beta}{1-\tau^{2}}(x-\tau) \geq 0 \quad(x \in[-1,1], \tau \in(-1,1)) .
$$

The left-hand side is linear in $x$, so we have to show the inequality only for $x=-1$ and $x=1$. For $x=-1$ we have

$$
1-\frac{(\alpha+\beta+2) \tau+\alpha-\beta}{1-\tau^{2}}(-1-\tau)=\frac{(\alpha+\beta+1) \tau+1+\alpha-\beta}{1-\tau}
$$

Now the numerator is again a linear function in $\tau$, and it takes the values $-2 \beta \geq 0$ for $\tau=-1$ and $2+2 \alpha>0$ for $\tau=1$, hence it is greather or equal to 0 for all $\tau \in(-1,1)$.

The case $x=1$ can be handled in the same way: We have

$$
1-\frac{(\alpha+\beta+2) \tau+\alpha-\beta}{1-\tau^{2}}(1-\tau)=\frac{1-\alpha+\beta-(\alpha+\beta+1) \tau}{1+\tau},
$$

and the numerator is $2+2 \beta>0$ for $\tau=-1$ and $-2 \alpha \geq 0$ for $\tau=1$

Naturally, this bound is sharp - the sum is equal to 1 for $x=\tau_{i}$.

\section{Explicit calculation of $\sum_{i=1}^{n} h_{i}^{2}(x)$ for Chebyshev polynomials}

The explicit calculation of this sum is possible for Chebyshev polynomials of first, second and third (or mixed) kinds. Our starting point is the interpolation identity from Lemma 2. The coefficients $c_{j}$ defined in Lemma 3 can be calculated from the differential equation (8) for any values of $\alpha$ and $\beta$. But we will see that for subsequent simplifications we need explicit (and simple) formulas for $p_{n}^{\prime}\left(\tau_{i}\right)$. This is possible only for Chebyshev polynomials.

Lemma 4. For any zero $\tau$ of $T_{n}, U_{n}, V_{n}$ we have

$$
\begin{aligned}
& \left(1-\tau^{2}\right) T_{n}^{\prime}(\tau)=\frac{n}{U_{n-1}(\tau)} \\
& T_{n}^{\prime 2}(\tau)=\frac{n^{2}}{1-\tau^{2}} \\
& \left(1-\tau^{2}\right) U_{n}^{\prime}(\tau)=\frac{n+1}{U_{n-1}(\tau)} \\
& \left(1-\tau^{2}\right) V_{n}^{\prime}(\tau)=\frac{n+\frac{1}{2}}{U_{n-1}(\tau)} \\
& U_{n}^{\prime 2}(\tau)=\left(\frac{n+1}{1-\tau^{2}}\right)^{2} \\
& V_{n}^{\prime 2}(\tau)=\frac{(2 n+1)^{2}}{2\left(1-\tau^{2}\right)(1+\tau)}
\end{aligned}
$$

respectively.

Proof. This follows immediately from the well-known trigonometric representation of Chebyshev polynomials. Differentiating $T_{n}(\cos \theta)=\cos n \theta$ we obtain

$$
\sin \theta T^{\prime}(\cos \theta)=n \sin n \theta \text {. }
$$


If $\cos \theta$ is a zero of $T_{n}$, then we have $\cos n \theta=0$ and consequently $\sin ^{2} n \theta=1$. This implies

$$
\left(1-\cos ^{2} \theta\right) T^{\prime}(\cos \theta)=\sin ^{2} \theta T^{\prime}(\cos \theta)=n \sin \theta \sin n \theta=\frac{n \sin \theta}{\sin n \theta}=\frac{n}{U_{n-1}(\cos \theta)} .
$$

From the above equation (15), by taking squares, we obtain

$$
\left(1-\cos ^{2} \theta\right) T^{\prime 2}(\cos \theta)=\sin ^{2} \theta T^{\prime 2}(\cos \theta)=n^{2} \sin ^{2} n \theta=n^{2},
$$

if $\cos n \theta=0$.

The derivation of the other equations is very similar. If $\cos \theta$ is a zero of $U_{n}$, then $\sin (n+1) \theta=0$ and consequently $\cos ^{2}(n+1) \theta=1$. From $\sin \theta U_{n}(\cos \theta)=\sin (n+1) \theta$ by differentiating we have the identity

$$
-\sin ^{2} \theta U_{n}^{\prime}(\cos \theta)+\cos \theta U_{n}(\cos \theta)=(n+1) \cos (n+1) \theta
$$

and by squaring both sides (using $U_{n}(\cos \theta)=0$ and $\cos ^{2}(n+1) \theta=1$ ) we obtain

$$
\left(1-\cos ^{2} \theta\right)^{2} U_{n}^{\prime 2}(\cos \theta)=(n+1)^{2} \text {. }
$$

From equation (16) we conclude

$$
\begin{aligned}
-\sin ^{2} \theta U_{n}^{\prime}(\cos \theta) & =(n+1) \cos (n+1) \theta=\frac{n+1}{\cos (n+1) \theta} \\
& =\frac{(n+1) \sin \theta}{\sin \theta \cos (n+1) \theta-\cos \theta \sin (n+1) \theta} \\
& =\frac{(n+1) \sin \theta}{-\sin n \theta}=-\frac{n+1}{U_{n-1}(\cos \theta)},
\end{aligned}
$$

where we used again $\cos ^{2}(n+1) \theta=1$ and $\sin (n+1) \theta=0$. Observing $\sin ^{2} \theta=1-\cos ^{2} \theta$ we arrive at our proposition.

The proof of the last equations relies on the trigonometric representation (14), and the fact that $\cos \left(n+\frac{1}{2}\right) \theta=0$ implies $\sin ^{2}\left(n+\frac{1}{2}\right) \theta=1$ and

$$
\sin \left(n+\frac{1}{2}\right) \theta=\frac{1}{\sin \left(n+\frac{1}{2}\right) \theta}
$$

(we omit details here)

Now we are able to formulate the main result of this section.

Theorem 3. In the case of Chebyshev polynomials of first kind ( $\left.\alpha=\beta=-\frac{1}{2}\right)$ the following formula holds:

$$
\sum_{i=1}^{n} h_{i}^{2}(x)=1-\left[\frac{2}{3}-\frac{1}{6 n^{2}}-\frac{1}{2 n^{2}} \cdot \frac{1-\frac{1}{2 n} x U_{2 n-1}(x)}{1-x^{2}}\right] T_{n}^{2}(x) .
$$


For Chebyshev polynomials of second kind $\left(\alpha=\beta=\frac{1}{2}\right)$ we have

$$
\begin{aligned}
\sum_{i=1}^{n} h_{i}^{2}(x)= & 1-\frac{1}{(n+1)^{2}}\left[\frac{7}{2} x^{2}+\frac{2}{3}\left(n^{2}+n-3\right)\left(1-x^{2}\right)\right] U_{n}^{2}(x) \\
& +\frac{1}{(n+1)^{3}}\left[\frac{9}{4} U_{n-2}(x)-\frac{5}{4} U_{n-4}(x)\right] U_{n}^{3}(x) .
\end{aligned}
$$

Finally, for Chebyshev polynomials of third kind $\left(\alpha=-\frac{1}{2}\right.$ and $\left.\beta=\frac{1}{2}\right)$ we find

$$
\begin{aligned}
\sum_{i=1}^{n} h_{i}^{2}(x)= & 1-\frac{1}{(2 n+1)^{2}}\left[-4 x+\frac{4}{3}\left(n^{2}+n\right)(x+1)-s_{n}(x)\right] V_{n}^{2}(x) \\
& +\frac{2}{(2 n+1)^{3}}\left[U_{n-2}(x)+U_{n-3}(x)\right] V_{n}^{3}(x)
\end{aligned}
$$

where

$$
s_{n}(x)=\frac{1+\frac{1}{2 n+1}\left[U_{n-2}(x)-3 U_{n-1}(x)\right] V_{n}(x)}{1-x},
$$

and $V_{n}(x)=U_{n}(x)-U_{n-1}(x)$.

Proof. As we can see easily, we have $h_{i}(x)=\left[1+\frac{1}{2} d_{1}\left(\tau_{i}\right)\left(x-\tau_{i}\right)\right] l_{i}^{2}(x)$ and thus, using Lemma 3 ,

$$
\begin{aligned}
h_{i}^{2}(x) & =b_{i}(x)+\left[\left(\frac{1}{4} d_{1}^{2}\left(\tau_{i}\right)-d_{2}\left(\tau_{i}\right)\right)\left(x-\tau_{i}\right)^{2}-d_{3}\left(\tau_{i}\right)\left(x-\tau_{i}\right)^{3}\right] l_{i}^{4}(x) \\
& =b_{i}(x)+p_{n}^{2}(x)\left[\left(\frac{1}{4} d_{1}^{2}\left(\tau_{i}\right)-d_{2}\left(\tau_{i}\right)\right)-d_{3}\left(\tau_{i}\right)\left(x-\tau_{i}\right)\right] \frac{1}{p_{n}^{\prime 2}\left(\tau_{i}\right)} l_{i}^{2}(x)
\end{aligned}
$$

for any set of orthogonal polynomials. Now we use our explicit formulas for $p_{n}^{\prime 2}\left(\tau_{i}\right)$ in the three special cases we consider.

Chebyshev polynomials of first kind: Computing the $c_{j}\left(\tau_{i}\right)$ from the differential equation (8), $d_{j}\left(\tau_{i}\right)$ from Lemma 3 , and observing $h_{i}(x)=\left[1-\tau_{i}\left(x-\tau_{i}\right) /\left(1-\tau_{i}^{2}\right)\right] l_{i}^{2}(x)$, we obtain after an easy (but tedious) calculation

$$
h_{i}^{2}(x)=b_{i}(x)+T_{n}^{2}(x)\left[\frac{1}{2 n^{2}} \cdot \frac{1}{1-\tau_{i}^{2}} l_{i}^{2}(x)-\left(\frac{2}{3}-\frac{1}{6 n^{2}}\right) h_{i}(x)\right] .
$$

From the above interpolation identities we know that

$$
\sum_{i=1}^{n} h_{i}(x)=\sum_{i=1}^{n} b_{i}(x) \equiv 1
$$

but we still have to evaluate the polynomial

$$
r_{n}(x)=\sum_{i=1}^{n} \frac{1}{1-\tau_{i}^{2}} l_{i}^{2}(x)
$$


The trick here is to calculate $\left(1-x^{2}\right) r_{n}(x)$, since we have

$$
\frac{1-x^{2}}{1-\tau_{i}^{2}} l_{i}^{2}(x)=\frac{1-\tau_{i} x}{1-\tau_{i}^{2}} l_{i}^{2}(x)-x \frac{x-\tau_{i}}{1-\tau_{i}^{2}} l_{i}^{2}(x)=h_{i}(x)-\frac{x T_{n}(x)}{n} U_{n-1}\left(\tau_{i}\right) l_{i}(x)
$$

(where we used $\left(x-\tau_{i}\right) l_{i}(x)=T_{n}(x) / T_{n}^{\prime}\left(T_{i}\right)$ and Lemma 4), and this gives immediately

$$
\left(1-x^{2}\right) r_{n}(x)=1-\frac{x T_{n}(x)}{n} U_{n-1}(x)=1-\frac{1}{2 n} x U_{2 n-1}(x) .
$$

Substituting this into the above equation, we obtain our first proposition.

Chebyshev polynomials of second kind: As above we compute the representation

$$
\begin{aligned}
h_{i}^{2}(x)= & b_{i}(x)+\frac{1}{(n+1)^{2}} U_{n}^{2}(x)\left\{\left[-\frac{7}{2} \tau_{i}^{2}-\frac{2}{3}\left(n^{2}+2 n-3\right)\left(1-\tau_{i}^{2}\right)\right]\right. \\
& \left.+\left[\frac{10 \tau_{i}^{3}}{1-\tau_{i}^{2}}+\left(\frac{10}{3} n^{2}+\frac{20}{3} n-\frac{15}{2}\right) \tau_{i}\right]\left(x-\tau_{i}\right)\right\} l_{i}^{2}(x) .
\end{aligned}
$$

The sum

$$
\sum_{i=1}^{n}\left[-\frac{7}{2} \tau_{i}^{2}-\frac{2}{3}\left(n^{2}+2 n-3\right)\left(1-\tau_{i}^{2}\right)\right] l_{i}^{2}(x)
$$

can be evaluated by equation (10), with $\alpha=\beta=\frac{1}{2}$ and $q(x)=-\frac{7}{2} x^{2}-\frac{2}{3}\left(n^{2}+2 n-\right.$ $3)\left(1-x^{2}\right)$, and this gives

$$
\sum_{i=1}^{n} h_{i}^{2}(x)=1+\frac{1}{(n+1)^{2}} U_{n}^{2}(x)\left\{q(x)+\sum_{i=1}^{n} \frac{\frac{19}{2} \tau_{i}-10 \tau_{i}^{3}}{1-\tau_{i}^{2}}\left(x-\dot{\tau}_{i}\right) l_{i}^{2}(x)\right\} .
$$

Now we can use $\left(x-\tau_{i}\right) l_{i}(x)=U_{n}(x) / U_{n}^{\prime}\left(\tau_{i}\right)$ and the formula for $U_{n}^{\prime}\left(\tau_{i}\right)$ from Lemma 4 to obtain

$$
\sum_{i=1}^{n} \frac{\frac{19}{2} \tau_{i}-10 \tau_{i}^{3}}{1-\tau_{i}^{2}}\left(x-\tau_{i}\right) l_{i}^{2}(x)=\frac{1}{n+1} U_{n}(x) \sum_{i=1}^{n}\left(\frac{19}{2} \tau_{i}-10 \tau_{i}^{3}\right) U_{n-1}\left(\tau_{i}\right) l_{i}(x) .
$$

Repeatedly applying the recurrence relation for the polynomials $U_{j}$ and observing $U_{n}\left(\tau_{i}\right)=0$, we can calculate

$$
\left(\frac{19}{2} \tau_{i}-10 \tau_{i}^{3}\right) U_{n-1}\left(\tau_{i}\right)=\frac{9}{4} U_{n-2}\left(\tau_{i}\right)-\frac{5}{4} U_{n-4}\left(\tau_{i}\right),
$$

and by the Lagrange interpolation formula we have

$$
\sum_{i=1}^{n}\left[\frac{9}{4} U_{n-2}\left(\tau_{i}\right)-\frac{5}{4} U_{n-4}\left(\tau_{i}\right)\right] l_{i}(x)=\frac{9}{4} U_{n-2}(x)-\frac{5}{4} U_{n-4}(x) .
$$

Chebyshev polynomials of third kind: Our starting point is again a representation

$$
h_{i}^{2}(x)=b_{i}(x)-\frac{V_{n}^{2}(x)}{(2 n+1)^{2}}\left[C_{0}\left(\tau_{i}\right)+C_{1}\left(\tau_{i}\right)\left(x-\tau_{i}\right)\right] l_{i}^{2}(x)
$$


where

$$
C_{0}(\tau)=-4 \tau+\frac{4}{3}\left(n^{2}+n\right)(\tau+1)+\frac{1}{\tau-1}
$$

and

$$
C_{1}(\tau)=\frac{2}{3} \frac{6\left(n^{2}+n-2\right) \tau^{2}+2\left(n^{2}+n+3\right) \tau-4 n^{2}-4 n+3}{\tau^{2}-1}
$$

are rational functions of $\tau$. The sum

$$
\sum_{i=1}^{n} q\left(\tau_{i}\right) l_{i}^{2}(x) \quad \text { with } \quad q(\tau)=-4 \tau+\frac{4}{3}\left(n^{2}+n\right)(\tau+1)
$$

can be calculated from formula (10) again. The sum on the right-hand side of (10) combines with $\sum_{i=1}^{n} C_{1}\left(\tau_{i}\right)\left(x-\tau_{i}\right) l_{i}^{2}(x)$ to give

$$
\begin{aligned}
\sum_{i=1}^{n} \frac{-4 \tau_{i}^{2}+2}{1-\tau_{i}^{2}}\left(x-\tau_{i}\right) l_{i}^{2}(x) & =\frac{V_{n}(x)}{n+\frac{1}{2}} \sum_{i=1}^{n}\left(-4 \tau_{i}^{2}+2\right) U_{n-1}\left(\tau_{i}\right) l_{i}(x) \\
& =\frac{V_{n}(x)}{n+\frac{1}{2}} \sum_{i=1}^{n}\left[-U_{n-3}\left(\tau_{i}\right)-U_{n-2}\left(\tau_{i}\right)\right] l_{i}(x) \\
& =\frac{V_{n}(x)}{n+\frac{1}{2}}\left[-U_{n-3}(x)-U_{n-2}(x)\right]
\end{aligned}
$$

(where we used Lemma 4 , the recurrence relation for $U_{j}$, and the fact that $U_{n}\left(\tau_{i}\right)=$ $\left.U_{n-1}\left(\tau_{i}\right)\right)$. However, we still have to calculate the sum

$$
s_{n}(x)=\sum_{i=1}^{n} \frac{1}{1-\tau_{i}} l_{i}^{2}(x) .
$$

We use the above trick to calculate $(1-x) s_{n}(x)$, since we have the identity

$$
\frac{1-x}{1-\tau_{i}} l_{i}^{2}(x)=h_{i}(x)+\frac{\tau_{i}-2}{1-\tau_{i}^{2}}\left(x-\tau_{i}\right) l_{i}^{2}(x) .
$$

With the same procedure as before we easily obtain the proposition.

This finally proves our theorem. We remark here that the simple but tedious algebraic manipulations were performed partially with MAPLE

Our last result will be the proof of an inequality conjectured by Gautschi already in his paper [6].

Corollary 2. In the case $d \sigma(x)=d x / \sqrt{1-x^{2}}$, the inequality $g_{n}(x) \leq 1$ holds for all $x \in[-1,1]$ and all $n \geq 2$.

Proof. As an immediate consequence of Theorem 3 and Corollary 1, we obtain the equation

$$
g_{n}(x)=1-\left[\frac{2}{3}-\frac{1}{6 n^{2}}-\frac{1}{2 n^{2}} r_{n}(x)-\frac{1}{\pi^{2}}\left\{1-x^{2}+\frac{3}{2 n} T_{n-2}(x) T_{n}(x)\right\}\right] T_{n}^{2}(x),
$$


where as in the proof of Theorem 3 we use the abbreviation

$$
r_{n}(x)=\sum_{i=1}^{n} \frac{1}{1-\tau_{i}^{2}} l_{i}^{2}(x)
$$

In order to estimate $r_{n}(x)$, we use another way to evaluate this sum. From equation (3) and Lemma 4 we have

$$
\frac{1}{1-\tau_{i}^{2}} l_{i}^{2}(x)=\frac{1}{n^{2}} \frac{T_{n}^{2}(x)}{\left(x-\tau_{i}\right)^{2}}=\frac{1}{n^{2}}\left[\frac{T_{n}(x)-T_{n}\left(\tau_{i}\right)}{x-\tau_{i}}\right]^{2} .
$$

Now the right-most expression is a polynomial in $\tau_{i}$ of degree $2 n-2$, and thus by virtue of the quadrature formula

$$
r_{n}(x)=\frac{1}{\pi n} \sum_{i=1}^{n} \sigma_{i}\left[\frac{T_{n}(x)-T_{n}\left(\tau_{i}\right)}{x-\tau_{i}}\right]^{2}=\frac{1}{\pi n} \int\left[\frac{T_{n}(x)-T_{n}(y)}{x-y}\right]^{2} d \sigma(y) .
$$

Using the identity

$$
\frac{T_{n}(x)-T_{n}(y)}{x-y}=U_{n-1}(x)+2 \sum_{k=1}^{n-1} U_{k-1}(x) T_{n-k}(y)
$$

(which can be derived easily from the generating functions of Chebyshev polynomials) and the orthogonality of $T_{n-k}$ with respect to $\sigma$, we obtain

$$
r_{n}(x)=\frac{1}{n}\left[U_{n-1}^{2}(x)+2 \sum_{k=1}^{n-1} U_{k-1}^{2}(x)\right] \text {. }
$$

This equation implies the inequality

$$
r_{n}(x) \leq \frac{1}{n}\left(n^{2}+2 \sum_{k=1}^{n-1} k^{2}\right)=\frac{2}{3} n^{2}+\frac{1}{3}
$$

Observing $T_{n-2}(x) T_{n}(x)=x^{2}-1+T_{n-1}^{2}(x) \leq x^{2}$ and thus

$$
1-x^{2}+\frac{3}{2 n} T_{n-2}(x) T_{n}(x) \leq 1-x^{2}+\frac{3}{2 n} x^{2} \leq 1,
$$

we see that the expression in square brackets in equation (17) is not less than

$$
\frac{1}{3}-\frac{1}{3 n^{2}}-\frac{1}{\pi^{2}} \geq \frac{1}{4}-\frac{1}{\pi^{2}}>0
$$

for $x \in[-1,1]$ and $n \geq 2$ 


\section{References}

[1] Abramowitz, M. and I. Stegun (Eds.): Pocketbook of Mathematical Functions. Thun Frankfurt/Main: Verlag Harri Deutsch 1984.

[2] Fischer, H.-J.: On the condition of orthogonal polynomials via modified moments. Z. Anal. Anw. 15 (1996), 223 - 244.

[3] Fischer, H.-J.: On generating orthogonal polynomials with respect to discrete measures. Z. Anal. Anw. 17 (1998), 183 - 205.

[4] Gautschi, W.: Questions of numerical condition related to polynomials. In: Recent Advances in| Numerical Analysis (eds.: C. de Boor and G. H. Golub). New York: Academic Press 1978, pp. $45-72$.

[5] Gautschi, W.: On generating orthogonal polynomials. SIAM J. Sci. and Stat. Comp. 3 (1982), $289-317$.

[6] Gautschi, W.: On the sensitivity of orthogonal polynomials to perturbations in the moments. Num. Math. 48 (1986), $369-382$.

[7] Gautschi, W.: On mean convergence of extended Lagrange interpolation. J. Comput. Appl. Math. 43 (1992), $19-35$.

[8] Gautschi, W.: Orthogonal polynomials: applications and computations. In: Acta Numerica 1996 (ed.: A. Iserles). Cambridge: Univ. Press 1986, pp. 45 - 119.

[9] Gautschi, W.: On mean convergence of extended Lagrange interpolation. J. Comput. Appl. Math. 43 (1992), $19-35$.

[10] Mason, J. C. and G. H. Elliott: Near-minimax complex approximation by four kinds of Chebyshev polynomial expansions. J. Comput. Appl. Math. 46 (1993), $291-300$.

[11] Mason, J. C.: Chebyshev polynomials of second, third and fourth kinds. J. Comput. A ppl. Math. 49 (1993), $169-178$. 